in the helium range ${ }^{1}$, so that the quenching of the orbital momentum should apply equally in the two cases. Owing to the closer approach of the ions, the magnetic interaction specific heat is naturally shifted to a higher temperature, but the most remarkable feature is the presence of a very large specific heat throughout the whole region below $1^{\circ} \mathrm{K}$. A first survey of the specific heat in the helium range indicated that it only falls to a low value at about $4.5^{\circ} \mathrm{K}$. The large extent of the maximum is remarkable and may have important applications in experimental technique. The specimen was an ellipsoid of axis ratio $3 \cdot 18: 1$, and consisted of very many small crystals of purity better than 99.9 per cent compressed by about 100 atmospheres into a coherent mass.

An attempt was made to estimate absolute temperatures by demagnetizing from different fields at the same bath temperature, and calculating the entropy of magnetization assuming the Brillouin formula and taking $j=\frac{1}{2}$. The calculation broke down for demagnetization from those fields which were so low that the level splitting was comparable with the energy of magnetization, but we may accept with caution the values for the lower temperatures produced by demagnetization from the higher fields. It appears probable that the susceptibility becomes extremely large at $0.05^{\circ} \mathrm{K}$., so that we might expect ferromagnetism near this temperature. The lowest point reached with copper sulphate was $0.08^{\circ} \mathrm{K} .\left(0.03^{\circ} T^{*}\right)$, but no hysteresis was observed with a ballistic galvanometer, though the arrangement was amply sensitive to detect the effect in ferric alum.

While it would be rash at this stage to attempt a detailed explanation, the general conclusion must be drawn that in copper sulphate there does exist a splitting of the energy levels such as to give a high specific heat in the helium range. Unless the effect is due to exchange forces, which seems improbable in view of the high dilution, it must be assumed that the electric field of the lattice can, in certain circumstances present in copper sulphate but absent in copper potassium sulphate, produce a level splitting even in an ion which is effectively in an ${ }^{2} S$ state owing to the quenching of its orbital momentum.

Royal Society Mond Laboratory,
Cambridge. March 29.

${ }^{1}$ Reekie, J., in the Press.

$$
\text { J. Ashmead. }
$$

\section{Sparking Potential of Deuterium (Heavy Hydrogen)}

THE sparking potential of heavy hydrogen between parallel electrodes was recently measured in this laboratory, and the influence of the material of the cathode was also examined. In accordance with the general theory of the discharge, the effect of a change of cathode material was most pronounced at the minimum sparking potential, which was found to be characteristic of the metal of the cathode after it had been de-gassed. Further, for any given metal the minimum sparking potential was found to be very sensitive to the state of the cathode surface even after it had been de-gassed in a vacuum furnace, as exposure of the cathode to the gas for any length of time had a marked effect in lowering the sparking potential.
The value of the minimum sparking potential of heavy hydrogen characteristic of a steel cathode was found to be 339 volts, as the experiments indicated that lower values, obtained after the metal had been exposed to the gas at pressures exceeding about $20 \mathrm{~mm}$. of mercury, were probably due to adsorbed layers of the gas on the cathode surface. However, in many cases, bombardment of the cathode by positive ions in a discharge was able to restore the higher values of the sparking potential. Similar effects were also observed with light hydrogen, and the minimum sparking potential with a steel cathode was found to be more than 10 per cent greater than that recently given for a platinum cathode ${ }^{1}$.

Hence the results show that typical diatomic gases like hydrogen and deuterium behave like the monatomic gases ${ }^{2,3}$ in that the minimum sparking potential is strongly characteristic of the cathode material, and increases with the work function of the cathode, a result which was not supported by the early measurements with the diatomic gases.

In comparing the two isotopes, it was found that for six different cathodes the minimum sparking potential of deuterium was greater than that of hydrogen by about 3 per cent, but that when the spark parameter $p d$ was about $5(\mathrm{~mm}$. of mercury $\times \mathrm{cm}$.) the sparking potential $V$ of the two gases was the same. The normal cathode fall is also known to be greater in deuterium than in hydrogen ${ }^{4}$. Now, it is reasonable to assume that the coefficient of ionization of deuterium by electrons is not very different from that of hydrogen, especially at high values of the ratio of $V / p d$, a view supported by the measured values of the force in the positive column ${ }^{5}$. On this assumption, then, the difference between the sparking potentials of the two gases can be accounted for if, at the same value of $V / p d$, the deuterium positive ion is 20 per cent less effective than the hydrogen ion in liberating electrons from the cathode, although both ions have the same potential energy. The difference must therefore be due to the lower velocity of the deuterium ion. Further, if this constitutes the main difference between the electrical properties of the two gases, it also accounts for the equality in the sparking potentials when $p d$ is about 5 .

Department of Physics, University College, Swansea. April 14.

${ }^{1}$ Ehrenkranz, Phys. Rev., 55, 219 (1939).

2 Llewellyn Jones and Galloway, NATure, 138, 973 (1936). Proc. Phys. Soc., 50, 207 (1938).

${ }^{3}$ Llewellyn Jones and Henderson, Phil. Mag., in the Press.

"Guntherschulze und Schnitger, Z. Phys., 103, 491 (1936).

s Steenbeck, Z. tech. Phys., 11, 397 (1936).

\section{Penetration of the Potential Barrier and Formation of $\mathrm{AlH}$}

Several investigators have observed peculiar anomalous intensities in the band system ${ }^{1} \mathbf{I I}-{ }^{1} \Sigma$ of the spectrum from AlH. Generally the lines from rotational levels $J>17$ in $v=0$ and $J>7$ in $v=I^{1} \mathrm{II}$ are weakened or absent according to the circumstances in the light-source.

In order to investigate whether formation of $\mathrm{AlH}$ takes place in the III-state, I have repeated an experiment of Wurm ${ }^{1}$, who investigated the spectrum of aluminium in a hydrogen atmosphere in an electric furnace. My spectrograms show that all 